\title{
INFLUÊNCIA DO EXERCÍCIO FÍSICO NA QUALIDADE DE VIDA DE IDOSAS DE UM GRUPO SOCIAL DE TAPEJARA, PR
}

\author{
Fábio Ricardo ACENCIO ${ }^{1}$ \\ Jéssica Bino GOMES ${ }^{2}$ \\ Mateus Dias ANTUNES ${ }^{3}$ \\ Glaukus Regiani BUENO ${ }^{4}$ \\ Gilberto Cezar PAVANELLI ${ }^{5}$
}

\begin{abstract}
${ }^{1}$ Profissional de Educação Física, discente do Programa de Pós Graduação em Promoção da Saúde do Centro Universitário de Maringá - UNICESUMAR (Bolsista Institucional) e docente da Universidade Paranaense (UNIPAR), Maringá, PR, Brasil. fabioricardo@unipar.br

${ }^{2}$ Profissional de Educação Física pela Universidade Paranaense - UNIPAR, Cianorte, PR, Brasil. jessicabinog@ hotmail.com

${ }^{3}$ Fisioterapeuta, discente do Programa de Pós Graduação em Promoção da Saúde do Centro Universitário de Maringá - UNICESUMAR (Bolsista CAPES), Maringá, PR, Brasil. mateus_antunes03 @ hotmail.com

${ }^{4}$ Fisioterapeuta, discente do Programa de Pós Graduação em Promoção da Saúde do Centro Universitário de Maringá - UNICESUMAR (Bolsista CAPES), Maringá, PR, Brasil. glaukusbueno@ hotmail.com

${ }^{5}$ Biólogo, docente do Programa de Pós Graduação em Promoção da Saúde, UNICESUMAR, Maringá, PR, Brasil. Pesquisador científico do CNPq, nível 1 e do Instituto Cesumar de Ciência, Tecnologia e Inovação (ICETI).gcpavanelli@pq.cnpq.br
\end{abstract}

Recebido em: 20/12/2016 - Aprovado em: 20/11/2017 - Disponibilizado em: 30/12/2017

\begin{abstract}
RESUMO
Objetivo: analisar a influência do exercício físico nas atividades de vida diária de idosas praticantes de um grupo social. Metodologia: estudo transversal com 40 idosas praticantes de exercícios físicos em um grupo social. Foi utilizado o teste de flexão de cotovelo e o de sentar e levantar da cadeira por 30 segundos da bateria de testes de Rikli e Jones que avalia as capacidades físicas. As idosas participantes do estudo eram pertencentes de um grupo social com três encontros semanais com duração de 1 hora que realiza exercícios físicos, dentre ele caminhada, hidroginástica e trabalhos de alongamento e fortalecimento muscular localizado no município de Jussara, no estado do Paraná. Os dados foram digitados em planilha do programa Microsoft Excel 2010. Os resultados foram apresentados por meio da estatística descritiva (média e desvio-padrão, tabelas e gráficos). Resultados: todos os valores obtidos no presente estudo foram superiores aos de referencia para cada faixa etária das idosas. Destaca-se no teste de flexão de cotovelo a média foi de $24 \pm 5,80$ e o esperado era de 15,2 para faixa etária de 65-69 anos. Já para o teste de sentar e levantar da cadeira o maior valor obtido foi na média referente da idade de 60 a 64 anos sendo 17 $\pm 2,31$ e o esperado era 14,5. Conclusão: Diante dos resultados obtidos, podemos observar que as idosas praticantes de exercícios físicos em um grupo social apresentam valores maiores na funcionalidade, sendo esta uma capacidade que atua diretamente nas atividades de vida diária do idoso.
\end{abstract}

Palavras-chave: Idoso, Atividade Motora, Força Muscular, Promoção da Saúde.

\begin{abstract}
Aim: to analyze the influence of physical exercise on the activities of daily life of elderly women practicing a social group. Methods: cross-sectional study with 40 elderly women practicing physical exercises in a social group. The elbow flexion test and the sit-and-lift test were used for 30 seconds of the Rikli and Jones test battery that evaluates the physical capacities. The elderly participants of the study belonged to a social group with three weekly encounters lasting 1 hour that performed physical exercises, among them walking, water aerobics and stretching and muscle strengthening works located in the municipality of Jussara, in the state of Paraná. The data were entered in a spreadsheet of the program Microsoft Excel 2010. The results were presented through descriptive statistics (mean and standard deviation, tables and graphs). Results: all values obtained in the present
\end{abstract}


study were higher than the reference values for each age group of the elderly. In the elbow flexion test, the mean was $24 \pm 5.80$ and the expected was 15.2 for the 65-69 age group. Already for the test of sit and lift of the chair the highest value obtained was in the average referent of the age of 60 to 64 years being $17 \pm 2.31$ and the expected one was 14.5. Conclusion: considering the results obtained, we can observe that the elderly practicing physical exercises in a social group have higher values in the functionality, being this a capacity that acts directly in the activities of daily life of the elderly.

Keywords: Aged, Motor Activity, Muscle Strength, Health Promotion.

\section{INTRODUÇÃO}

A população idosa no Brasil vem aumentando de maneira acentuada nos últimos anos. A partir dos anos 40 as maiores taxas de crescimento ocorreram nas faixas etárias dos 60 anos. Se esta taxa se mantiver nesse patamar, em 2020 o país atingirá cerca de 31 milhões de idosos, número impensável há vários anos. Isso pode ser explicado pelo fato de há algum tempo os idosos terem buscado de maneira mais intensa melhoras na sua qualidade de vida, procurando por orientações médicas preventivas e terapêuticas, melhorando ainda sua socialização com familiares e a comunidade, além de intensificarem a prática de atividades físicas, entre outras ações. Essa mudança de comportamento fez com que aumentasse a expectativa de vida das pessoas dessa faixa etária (MARI et al., 2016, GUINATTI; ZAMAI, 2016).

Em relação a atividade física, devese destacar que que essa prática contribui cada vez mais para a melhora da saúde e bem-estar, refletindo diretamente na qualidade de vida dos idosos. Isso repercute diretamente no grau de autonomia das pessoas, que passam a realizar com mais facilidade tarefas do cotidiano como vestir uma roupa, varrer uma casa, lavar uma louça, ou seja, permite uma maior independência desses idosos nessas simples atividades rotineiras, mas importantes para facilitar seu dia a dia e aumentar sua autoestima (TORQUATO et al., 2016, SPOSITO; NERI; YASSUDA, 2016).

Assim, vários autores descrevem que parte da população já aceita naturalmente a importância da prática atividade física na terceira idade, em diferentes modalidades. Outros destacam a influência benéfica que essa prática produz na adaptabilidade e funcionalidade dos vários sistemas orgânicos dos idosos (BETA et al., 2016; QUEIROZ et al., 2016; SOUZA et al., 2016, SILVA et al., 2016)).

Um dos primeiros fatores a se manifestar nos adultos é a perda da massa magra e a consequente diminuição da força muscular. Isso é um fenômeno natural e a consequência é sua ação direta na perda de 
parte de seu rigor físico, o que o atrapalha na realização de diversas tarefas de seu cotidiano (BERTON et al., 2016).

Esse desgaste natural do sistema muscular dos idosos pode ser parcialmente compensada com os exercícios, que irão facilitar seus deslocamentos e movimentações, bem como diminuir as quedas que são muito frequentes nas pessoas idosas (ANJOS et al., 2015). Desta forma o presente estudo teve como objetivo analisar a influência do exercício físico nas atividades de vida diária de idosas praticantes de um grupo social.

\section{METODOLOGIA}

Para a realização desse estudo transversal valeu-se de idosas participantes de um grupo social localizado no município de Jussara - PR. Foram incluídas ao estudo pessoas idosas na faixa etária de 60 a 79 anos, pertencente ao grupo há no mínimo seis meses no grupo. Como critério de exclusão adotou-se o déficit neurológico, cognitivo e motor ou aqueles que estavam realizando outro tipo de intervenção.

Foi utilizado o teste de flexão de cotovelo, onde o avaliado permanecia sentado em uma cadeira, com as costas retas no encosto e pés totalmente apoiado no chão, com o lado dominante do corpo perto da extremidade lateral da cadeira. $\mathrm{O}$ peso era segurado de lado com a mão dominante fechada. O teste começava com braço estendido para baixo ao lado da cadeira, perpendicular ao chão. Ao sinal “Atenção! Já!” o avaliado vira a palma da mão para cima enquanto flexiona o braço, completando totalmente o ângulo de movimento, voltando depois à posição inicial com o cotovelo totalmente estendido. Ao retornar à posição, o peso deverá ser segurado com a mão fechada (RIKLI et al. JONES, 2001).

Para o teste de levantar da cadeira em 30 segundos o idoso inicia na posição sentada no meio da cadeira, com as costas retas e os pés apoiados no chão. Os braços ficam cruzados contra o tórax. Ao sinal “Atenção! Já!” o avaliado se levanta, ficando totalmente em pé e então retorna a uma posição completamente sentada. O avaliado é encorajado a sentar-se completamente o maior número possível de vezes em 30 segundos (RIKLI et al. JONES, 2001).

A tabela 1 apresenta valores padrões de ambos os sexos para a população feminina segundo a idade.

Tabela 1. Valores padrões de referência (repetições) para a população feminina dos testes de Rikli e Jones segundo a idade.

\begin{tabular}{|c|c|c|c|c|}
\hline \multirow[t]{2}{*}{ Testes } & \multicolumn{2}{|c|}{ IDADE } & \multirow[b]{2}{*}{$70-74$} & \multirow[b]{2}{*}{ 75-79 } \\
\hline & $60-64$ & 65-69 & & \\
\hline TFC & 16,1 & 15,2 & 14,5 & 14,0 \\
\hline TSLC & 14,5 & 13,5 & 12,9 & 12,5 \\
\hline
\end{tabular}

Legenda: TFC: Teste Flexão de Cotovelo; TSLC: Teste de Sentar e Levantar da cadeira. 
As idosas pertenciam a um grupo social que realizava três encontros semanais com duração de uma hora, onde realizavam vários exercícios físicos, destacando-se caminhada, hidroginástica, além de trabalhos de alongamento e fortalecimento muscular.

As idosas foram informadas quanto à justificativa, objetivos e procedimentos para a pesquisa com seres humanos constantes na Resolução 196/96 do Conselho Nacional de Saúde, após assinarem o Termo de Consentimento Livre e Esclarecido. O estudo foi aprovado pelo Comitê de Ética em Pesquisa Envolvendo Seres Humanos da Universidade Paranaense, por meio do parecer $\mathrm{n}^{\circ} 911.927$.

Os dados foram digitados em planilha do programa Microsoft Excel 2010. Os resultados foram apresentados por meio da estatística descritiva (média e desvio-padrão, tabelas e gráficos).

\section{RESULTADOS E DISCUSSÃO}

Foram avaliados 40 indivíduos do sexo feminino, com idades de 60 a 89 anos residentes na cidade de Jussara, PR. A Tabela 2 apresenta a média e o desvio padrão da idade das idosas em ambos os testes agrupados pela faixa etária.
Tabela 2. Valores da média e desvio padrão da idade das idosas agrupadas pela faixa etária nos testes de Rikli e Jones segundo a idade em Jussara, PR.

\begin{tabular}{lcccc}
\hline Testes & \multicolumn{4}{c}{ IDADE } \\
\cline { 2 - 5 } & $\mathbf{6 0 - 6 4}$ & $\mathbf{6 5 - 6 9}$ & $\mathbf{7 0 - 7 4}$ & $\mathbf{7 5 - 7 9}$ \\
\hline TFC & $23 \pm 4,80$ & $24 \pm 5,80$ & $21 \pm 6,51$ & $20 \pm 3,13$ \\
TSLC & $17 \pm 2,31$ & $14 \pm 3,23$ & $13,5 \pm 2,52$ & $13 \pm 2,30$ \\
\hline
\end{tabular}

Legenda: TFC: Teste Flexão de Cotovelo; TSLC: Teste de Sentar e Levantar da cadeira.

Tabela 3. Valores padrões de referência (repetições) para a população feminina dos testes de Rikli e Jones segundo a idade.

\begin{tabular}{lcrrr}
\hline \multirow{1}{*}{ Testes } & \multicolumn{3}{c}{ IDADE } & \\
\cline { 2 - 5 } & $\mathbf{6 0 - 6 4}$ & $\mathbf{6 5 - 6 9}$ & $\mathbf{7 0 - 7 4}$ & $\mathbf{7 5 - 7 9}$ \\
\hline TFC & 16,1 & 15,2 & 14,5 & 14,0 \\
TSLC & 14,5 & 13,5 & 12,9 & 12,5
\end{tabular}

Legenda: TFC: Teste Flexão de Cotovelo; TSLC: Teste de Sentar e Levantar da cadeira.

Todos os valores obtidos no presente estudo foram superiores aos de referencia para cada faixa etária das idosas. Destaca-se no teste de flexão de cotovelo a média foi de $24 \pm 5,80$ e o esperado era de 15,2 para faixa etária de 65-69 anos. Já para o teste de sentar e levantar da cadeira o maior valor obtido foi na média referente da idade de 60 a 64 anos sendo $17 \pm 2,31$ e o esperado era 14,5 .

Envelhecer com saúde traz benefícios não só ao indivíduo, mas também a sociedade. Quando a pessoa se torna idoso, é sabido que suas capacidades físicas já não são mais as mesmas (VERAS, 2016). Entretanto, apesar de todos terem o direito de manter-se bem, saudável e buscar por melhoras no seu condicionamento físico, isso nem sempre acontece(OLIVEIRA et al., 2016). 
Para Matsudo, Matsudo e Barros Neto (2001), a atividade física deve ser estimulada em qualquer etapa da vida, mesmo iniciando após os 50 anos. Isso serve como forma de prevenir e controlar as doenças crônicas, não transmissíveis que aparecem mais frequentemente durante a terceira idade e como forma de manter a independência funcional.

Nas sociedades industrializadas, as atividades físicas cada vez mais, representam um fator de qualidade de vida. Um estilo de vida mais ativo está associado a maior capacidade de trabalho físico e mental, mais entusiasmo para a vida e sensação de bem estar. No entanto, as atividades físicas realizadas pelo idoso devem ser moderadas e seguir um programa (ROMA et al., 2013).

As atividades que devem ser mais estimuladas são as aeróbicas de baixo impacto junto com os exercícios com pesos, para estimular a manutenção da força muscular dos membros superiores e inferiores, devendo ter prioridade no idoso (HAUSER et al., 2015).

A força é uma capacidade física de extrema importância na vida das pessoas, pois necessitamos dela para podermos realizar varias coisas em nosso cotidiano, como andar, levantar, sentar, vestir-se, entre outras (AGUIAR et al,, 2014). Vários estudos comprovam que o homem atinge sua força máxima entre 20 e 30 anos. A partir daí, inicia-se a perda gradativa da força (MONTEIRO et al., 2012; TOURINHO FILHO et al., 2013).

Ao compararmos os resultados do teste com a tabela de referência, verifica-se que os do presente estudo apresentaram valores mais altos do que os valores usados como referência.

Isto mostra um resultado positivo para esse grupo social de idosos que praticam frequentemente exercícios físicos. Assim, percebe-se a importância e necessidade de se praticar atividade física, pois além de tornar pessoas mais ativas, aumentar seu bem estar, possibilita melhoras em seu condicionamento físico. (SILVA et al., 2016).

Para Okuma (1998), os benefícios da atividade física são evidentes igualmente para o domínio das capacidades cognitivas e psicossociais. Reconhece-se sua forte relação com bem-estar psicológico, comumente indicado por sentimentos de satisfação, felicidade e envolvimento. Sabe-se que também pessoas que estão seguras de que dispõem das competências necessárias para um adequado funcionamento intelectual, físico, afetivo e social, ou seja, que sentem eficazes são beneficiadas no que tange à autoestima e aos motivos de realização (VERAS, 2016).

A prática de atividade física vem sendo citada em varias pesquisas como uns 
dos componentes mais importantes para uma boa qualidade de vida (SILVA et al, 2016). Pois atualmente homens e mulheres entre todas as idades estão buscando cada vez mais pela pratica da atividade física, com objetivo de buscar por melhoras no seu condicionamento físico e aumentar sua qualidade de vida (ZAMAI; BANKOFF; PERES, 2016; ZAMAI, 2016).

Para Terra e Dorneles (2003) a prática regular de atividade física, quando bem orientada, pode ocasionar maior longevidade, e diminuir taxa de morbidade e mortalidade, redução do uso de medicamentos prescritos, melhora da capacidade fisiológica em portadores de doenças crônicas, previne o declínio cognitivo, redução de frequências de quedas, manutenção da independência, e autonomia e benefícios psicológicos, como a melhoria do contato social e prazer pela vida.

De acordo com Campos (2002), para o cidadão comum enfrentar seu cotidiano e manter-se saudável, é recomendado que suas atividades físicas fossem orientadas para aumentar a tonicidade dos músculos, para torna-los mais fortalecidos. $\mathrm{O}$ autor completa dizendo que aqueles que levam uma vida sedentária e diminui suas atividades corporais perdem massa muscular, e consequentemente perde também a sua força.
Para Porto (2008), um estilo de vida mais ativo e a inclusão da pratica da atividade física no cotidiano do idoso, causa alterações nos componentes da aptidão física que se relacionam com a saúde, tais como a força muscular, a resistência muscular, flexibilidade. Tais alterações irão influenciar positivamente na execução das atividades da vida diária de forma segura e com autonomia.

Enfim, o idoso que busca pela prática da atividade física em seu cotidiano, mostra evidências convincentes, que grandes benefícios tanto na saúde como na manutenção do condicionamento físico, como também no seu bem-estar, é adquirida através da mesma. Pois além de grandes benefícios já citados, diminui a sua dependência e prolonga a qualidade e a duração de vida do individuo (FERRETTI et al., 2015).

Há um declínio natural no desempenho das capacidades físicas de acordo com o envelhecimento. Isto pode fazer com que alguns idosos se auto definam como indivíduos inválidos ao meio da sociedade, por não conseguirem se habituar as limitações que ocorre com o tempo. Isto pode influenciar no comportamento social, e normalmente esses indivíduos se excluem dos ambientes que frequentavam. Neste sentido, o mesmo pode ter prejuízo em sua saúde, como 
depressão, hipertensão e doenças degenerativas (WEINECK, 2003)

No entanto este estudo apresentou diversos meios pelo qual essa situação pode ser revertida, pois o hábito de atividade física, desde que orientado corretamente deve ser o objetivo de qualquer população, seja homem mulher, jovem, adulto ou idoso (LINO et al., 2015).

A inatividade pode ocorrer comprometimento as atividades da vida diária do idoso, que não pratica nenhuma atividade física. Pode dificultar as atividades mais simples, como vestir-se, subir degraus, caminhar, agachar, varrer, fazendo com que essas pessoas se tornem cada vez mais dependentes do próximo (BIANCHI et al., 2016).

Atualmente não precisa ter recursos financeiros para começar a se tornar uma pessoa mais ativa, projetos onde engloba toda a sociedade, vêm sendo oferecidos sem custo algum às pessoas, sendo dos mais jovens até a população de idosos (SILVA et al., 2015).

\section{CONCLUSÃO}

Diante dos resultados obtidos, podemos observar que as idosas praticantes de exercícios físicos em um grupo social apresentam valores maiores na funcionalidade, sendo esta uma capacidade que atua diretamente nas atividades de vida diária do idoso.

\section{REFERÊNCIAS}

AGUIAR, Pedro de Paula Leite et al. Avaliação da influência do treinamento resistido de força em idosos. Kairós Gerontologia, v. 17, n. 3, p. 201-217, 2014.

ANJOS, Fabiana Ribeiro dos et al. Probabilidade de cair e medo de quedas após oficina de equilíbrio em idosos praticantes de atividade física. Revista de Atenção à Saúde, v. 13, n. 44, p. 5-10, 2015.

BERTON, Ricardo et al. Influência da força muscular no volume e na intensidade da atividade física diária de idosos.

\section{Revista Brasileira de Educação Física e} Esporte, v. 30, n. 3, p. 541-546, 2016.

BÊTA, Fernanda Cristina Oliveira et al. Comparação dos efeitos do treinamento resistido e da hidroginástica na autonomia de indivíduos idosos. Revista Brasileira de Prescrição e Fisiologia do Exercício, v. 10, n. 58, p. 220-224, 2016.

BIANCHI, Adriane Behring et al. Estudo comparativo entre os métodos Pilates no solo e Water Pilates na qualidade de vida e dor de pacientes com lombalgia. Cinergis, v. 17, n. 4,p. 282-286, 2016.

CAMPOS, M.V; et. al. Atividade física passo a passo - saúde sem medo e sem preguiça. Brasília: Thesaurus, 2002. 248 p. il.

FERRETTI, Fátima et al. Análise da qualidade de vida em idosos praticantes e 
não praticantes de exercício físico regular.

\section{Estudos Interdisciplinares sobre o}

Envelhecimento, v. 20, n. 3, p. 729-743, 2015.

GUINATTI, Gabriela; ZAMAI, Carlos Aparecido. Consciência corporal e alongamento: contribuições para a melhoria da saúde e da qualidade de vida dos participantes do programa mexa-se. Sínteses: Revista Eletrônica do SIMTEC, v. 1, n. 3, p. 277-277, 2016.

HAUSER, Eduardo et al. Medo de cair e desempenho físico em idosos praticantes de atividade física. Revista da Educação Física, v. 26, n. 4, p. 593-600, 2015.

LINO, Ayala Sabino et al. Comparação do perfil lipídico e protéico entre adultos sedentários e idosos ativos em uma população selecionada da cidade de PatosPB. Informativo, v. 9, n. 1, p. 86-90, 2015.

MARI, Fernanda Rigoto et al. O processo de envelhecimento e a saúde: o que pensam as pessoas de meia-idade sobre o tema. Revista Brasileira de Geriatria e Gerontologia, v. 19, n. 1, p. 35-44, 2016.

MATSUDO, Sandra Mahecha; MATSUDO, Victor Keihan Rodrigues; BARROS NETO, Turíbio Leite. Atividade física e envelhecimento: aspectos epidemiológicos. Revista Brasileira de Medicina do Esporte, v. 7, n. 1, p. 2-13, 2001.

MONTEIRO, Walace D. et al. Força muscular e características morfológicas de mulheres idosas praticantes de um programa de atividades físicas. Revista Brasileira de Atividade Física \& Saúde, v. 4, n. 1, p. 20-28, 2012.
OKUMA,S.S. O idoso e a atividade física: fundamentos e pesquisa.

Campinas, SP: Papirus, 1998.

OLIVEIRA, Jéssica Heloisa dos Santos et al. Envelhecer com qualidade: reflexo de ações extensionistas em instituições asilares. Revista Ciência em Extensão, v. 12, n. 2, p. 141-152, 2016.

\section{PORTO. J.C. Longevidade: atividade}

física e envelhecimento. Maceió:

EDUFAL. 2008. 265 p. il.

QUEIROZ, Daiane B. Borges et al. Funcionalidade, aptidão motora e condições de saúde em idosos longevos residentes em domicílio. Arquivos de Ciências da Saúde, v. 23, n. 2, p. 47-53, 2016.

RIKLI, Roberta E.; JONES, C. Jessie. Senior fitness test. Champaign (IL): Human Kinetics, 2001.

ROMA, Maria Fernanda Bottino et al. Effects of resistance training and aerobic exercise in elderly people concerning physical fitness and ability: a prospective clinical trial. Einstein, v. 11, n. 2, p. 153157, 2013.

SILVA, Jéssica Andrade da et al. Efeito de um programa de exercício de 8 semanas na pressão arterial e gastos com saúde.

Pesquisa em Educação Ambiental, v. 2, n. 1, p. 1-4, 2015.

SILVA, Patrick Leonardo Nogueira da et al. Avaliação da qualidade de vida de idosos praticantes de atividade física de uma unidade básica de saúde de Minas Gerais. Revista da Universidade Vale do Rio Verde, v. 14, n. 2, p. 24-35, 2016.

SILVA, Rodrigo Silvino et al. Qualidade de vida e capacidade funcional de idosas 
praticantes de hidroginástica no município de Sarandi/PR. Revista Brasileira de Qualidade de Vida, v. 8, n. 1,p. 28-41, 2016.

SOUZA, Lara A. et al. Efeitos de um treino multissensorial supervisionado por seis semanas no equilíbrio e na qualidade de vida de idosos. Medicina, v. 49, n. 3, p. 223-231, 2016.

SPOSITO, Giovana; NERI, Anita Liberalesso; YASSUDA, Mônica Sanches. Atividades avançadas de vida diária (AAVDs) e o desempenho cognitivo em idosos residentes na comunidade: Dados do Estudo FIBRA Polo UNICAMP. Revista Brasileira de Geriatria e Gerontologia, v. 19, n. 1, p. 7-20, 2016.

TERRA, N.L; DORNELLES, B. Envelhecimento bem-sucedido. Porto Alegre: EDIPUCRS. $2^{\circ}$ ed. 2003.

TORQUATO, Edna et al. Comparação do nível de atividade física medido por acelerômetro e questionário IPAQ em idosos. Revista Brasileira de Atividade Física \& Saúde, v. 21, n. 2, p. 144-153, 2016.

TOURINHO FILHO, Hugo et al. Efeitos agudos do treinamento aeróbio sobre o desempenho da força muscular. Pensar a Prática, v. 16, n. 2, p. 320-327, 2013.
VERAS, Renato. É possível, no Brasil, envelhecer com saúde e qualidade de vida?. Revista Brasileira de Geriatria e Gerontologia, v. 19, n. 3, p. 381-382, 2016.

WEINECK, J. Treinamento ideal: instruções técnicas sobre o desempenho fisiológico, incluindo considerações específicas de treinamento infantil e juvenil. Manole. $9^{\circ}$ ed. 2003.

William Cordeiro et al. Exercício físico na promoção da saúde na terceira idade.

Saúde e Meio Ambiente: Revista

Interdisciplinar, v. 4, n. 1, p. 55-65, 2015

ZAMAI, Carlos Aparecido. Contribuições da prática de atividades físicas e laborais na melhoria da saúde e qualidade de vidaprograma mexa-se EMBRAPA. Sínteses, v. 4, n. 4, p. 196-196, 2016.

ZAMAI, Carlos Aparecido; BANKOFF, Antonia Dalla Pria; PERES, Cláudia Maria. Programa de convivência e atividade física "Mexa-se Unicamp": experiência bem sucedida na promoção da saúde da população universitária. Revista Saúde e Meio Ambiente, v. 1, n. 1, p. 55$65,2016$. 